\title{
Structure and relaxation in liquid and amorphous selenium
}

\author{
D. Caprion and H. R. Schober \\ Forschungszentrum Jülich, IFF, D-52425 Jülich, Germany
}

(Received 31 January 2000)

\begin{abstract}
We report a molecular dynamics simulation of selenium, described by a three-body interaction. The temperatures $T_{g}$ and $T_{c}$ and the structural properties are in agreement with experiment. The mean nearest neighbor coordination number is 2.1. A small prepeak at about $1 \AA^{-1}$ can be explained in terms of void correlations. In the intermediate self-scattering function, i.e., the density fluctuation correlation, classical behavior, $\alpha$ and $\beta$ regimes, is found. We also observe the plateau in the $\beta$ regime below $T_{g}$. In a second step, we investigated the heterogeneous and/or homogeneous behavior of the relaxations. At both short and long times the relaxations are homogeneous (or weakly heterogeneous). In the intermediate time scale, lowering the temperature increases the heterogeneity. We connect these different domains to the vibrational (ballistic), $\beta$ and $\alpha$ regimes. We have also shown that the increase in heterogeneity can be understood in terms of relaxations.
\end{abstract}

\section{INTRODUCTION}

Although glass is one of the most common materials, the glass transition is still poorly understood. It is a continuous transition in which the viscosity of the glass forming liquid increases from $10^{-3}$ Pas in the liquid to more than $10^{9}$ Pas in the supercooled state. It is, therefore, easy to understand that very different time scales become important near the glass transition, and different types of relaxations might be observed.

The mode coupling theory $(\mathrm{MCT})^{1}$ gives a microscopic picture of this transition. This theory focuses on the density correlation function $\Phi(q, t)$, the intermediate self-scattering function, and proposes a mechanism of backflow to explain the increase of the viscosity and/or of the time scales. ${ }^{2}$ One of the most striking results of the MCT is the prediction of a critical temperature $T_{c}$ below which the system becomes nonergodic. In other words the system is trapped in a well of the energy landscape. This feature is related to a nonzero value of $\Phi(q, t)$ when $t \rightarrow \infty$. Above $T_{c}$, the function $\Phi(q, t)$ shows a short time relaxation, related to the vibrational (often called ballistic) regime, and a long time one, also called $\alpha$ relaxation. Below $T_{c}$, a third regime appears, the so-called $\beta$ regime, which is first seen as a shoulder and saturates at a finite value below $T_{g}$.

This nonergodicity has been seen in many experimental measurements $^{3-6}$ and computer simulations ${ }^{7-10}$ on different types of materials ranging from the fragile polymeric glasses to strong glasses, such as $\mathrm{SiO}_{2}$. In this paper we want to go a step further. Using a model of selenium, we check for the presence of these two or three (depending on the temperature) relaxation steps, and ask the following question. Does each atom have the same probability of relaxing? If below $T_{c}$ the system becomes nonergodic, and is trapped in a well of the energy landscape, are all atoms still equivalent, or are some more (or less) mobile than others? We can reformulate this question and ask whether the relaxations are homogeneous or heterogeneous. According to some authors ${ }^{11,12}$ the relaxations should be more homogeneous, in particular in the $\alpha$ regime, whereas others ${ }^{13-21}$ claim that the relaxations in amorphous or disordered materials are more heterogeneous.
The answer therefore does not seem to be obvious.

From the theoretical point of view, simple one-atomic systems such as soft or hard spheres or Lennard-Jones systems would be optimal to study. Unfortunately these simple systems crystallize rapidly at temperatures near the glass transition temperature $T_{g}$ and, therefore, can be utilized only for studies in the liquid, well above $T_{g}$, or deep in the glassy state $T \ll T_{g}$. One possibility for avoiding crystallization is to introduce special features in the interatomic interaction potential which penalizes ordering. ${ }^{22,23}$ The most common approach is to use binary mixtures of atoms. A different approach is to simulate a real monoatomic glass former, such as selenium, which has been studied extensively in experiment (see the reviews ${ }^{24,25}$ ). Se is covalently bound and prefers a coordination number of two. This is reflected in the different crystal structures. The most stable trigonal phase consists of parallel helical chains. Two monoclinic forms are composed of rings of eight atoms. The polymorphs are distinguished by the correlation between neighboring dihedral angles. Depending on this correlation one has either a trans (chains) or a cis configuration (rings). The energy difference between the cis and trans configuration was estimated to be only $0.03 \mathrm{eV} .^{26}$ This low energy difference implies that in glasses both configurations should coexist, which in turn strongly hinders crystallization. From a first-principles molecular dynamics simulation using 64 atoms, Hohl and Jones $^{27}$ conclude that both amorphous and liquid selenium can be viewed as consisting of branched chains which include rings of different length. The small size prevented quantitative statistics of chain and ring lengths and branching points. The fraction of atoms having twofold coordination varies in the literature between $95 \%$ and $71 \% .^{27-30}$

To study dynamical properties, larger systems are needed and one has to resort to effective interatomic interactions. This immediately leads to the problem of simultaneously having to describe the covalent binding in the chains and rings and the van der Waals like interaction between the rings, as well as possible branching and bond breaking. One possibility is to disregard the last two, and to use different interactions for atoms in the same chain and in different chains, respectively. Similar to simulations of polymers one 
then considers a glass or a liquid of chains of a predefined length. This fragmented chain method has been employed both for electronic structure calculations ${ }^{31}$ and for classical molecular dynamics simulations. ${ }^{32,33}$ Another possibility is to use a simpler description of the electronic properties, such as tight binding models. ${ }^{28}$

We follow a different line using one effective interatomic potential for both the intrachain and the interchain interactions, as used previously by Stillinger, Weber, and LaViolette, in their study of liquid sulfur. ${ }^{34}$

This paper is laid out as follows: In Sec. II we report the details of the simulations, and of the production of the liquid and glassy samples used in the measurements of the different properties reported in this work. Section III is devoted to the determination of the glass transition temperature $T_{g}$ and the critical temperature $T_{c}$. Given these temperatures, we report the evolution of the structure through the glass transition in Sec. IV. In Sec. V we focus on the relaxations and the intermediate self-scattering function. Section VI presents the tools used to study the homogeneity or heterogeneity of these relaxations, reports the measurements, and discusses them. Finally we conclude in Sec. VII.

\section{SIMULATIONS}

We performed classical molecular dynamics simulations on a system of $N=2000$ atoms interacting via a three-body potential. This potential was built to reproduce the properties of small clusters of selenium and of the trigonal crystalline phase. Details of the potential are given in Ref. 35. The potential has previously been used to calculate the vibrations ${ }^{36}$ and local relaxations in amorphous Se. ${ }^{37}$ In these simulations it was shown that both the low frequency resonant vibrations and the local relaxations are centered on groups of ten and more atoms. This finding is in agreement with the interpretation of experiments by the soft potential model. ${ }^{38}$ From a Monte Carlo study of liquid Se it was concluded that the model provides a sound basis for the study of both the microscopic and the electronic structure, despite some deficiency in the treatment of the van der Waals interaction. ${ }^{39}$

During the simulations the pressure was fixed to $0 \mathrm{~Pa}$, i.e., we worked with equilibrium structures. In order to keep the pressure constant we used a Parrinello-Rahman algorithm. ${ }^{40,41}$ The temperature was kept constant by rescaling the velocities at each integration step. We determined that the way we control the pressure and temperature influenced neither the dynamics of the system nor the results of our simulations.

The equations of motion were integrated using the velocity Verlet algorithm. ${ }^{42}$ Taking care of the stability of the algorithm, we chose the time steps equal to $1 \mathrm{fs}$ in the liquid, $2 \mathrm{fs}$ in the glassy phase, and $4 \mathrm{fs}$ for the lowest temperature (6K).

To improve the statistics we used four independent starting configurations to obtain the different samples used in the measurement. These samples were produced as follows: first we equilibrated a liquid at $550 \mathrm{~K}$ (above the melting point), and cooled it to $290 \mathrm{~K}$ with a quench rate of $10^{13} \mathrm{~K} / \mathrm{s}$. At this temperature we let the systems equilibrate for $8 \mathrm{~ns}$ and then quenched them to $0 \mathrm{~K}$ with the same quench rate. Dur-

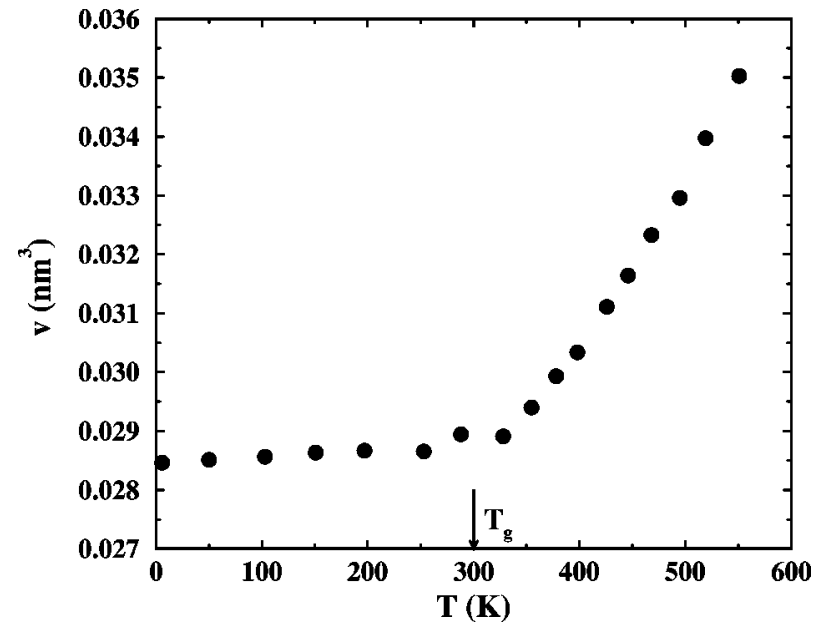

FIG. 1. Evolution of the atomic volume of liquid and amorphous Se atoms during the quench. The change of slope between high and low temperatures determines the glass transition temperature $T_{g}$.

ing both quenches we saved configurations at several temperatures and again equilibrated them before using them as input of the measurements. The equilibration times were $8 \mathrm{~ns}$ above $290 \mathrm{~K}, 16 \mathrm{~ns}$ between 290 and $6 \mathrm{~K}$, and $32 \mathrm{~ns}$ at $6 \mathrm{~K}$. After the equilibration period several relevant physical quantities were computed.

\section{III. $T_{g}$ AND $T_{c}$ DETERMINATION}

To obtain the relevant temperature scale, we first determined the glass transition temperature $T_{g}$. For this we followed the evolution of the volume during the quench process. As the coefficient of volume expansion is higher in the liquid than in the solid phase, one observes a change of slope of the volume curve when the system is quenched through the glass transition. From Fig. 1 the glass transition temperature is estimated as $T_{g} \approx 300 \mathrm{~K}$. Experimentally $T_{g}$ is about $305 \mathrm{~K}^{43}$ The good agreement between the simulated and experimental values of $T_{g}$ might seem surprising regarding the high quench rate and the usual discrepancies observed in simulation. However, one should note that due to the aging over several nonoseconds the effective quench rate is lower, $\approx 10^{10} \mathrm{~K} / \mathrm{s}$.

Another characteristic temperature is the critical temperature $T_{c}$ given by the MCT. This temperature can be obtained from the diffusion constant $D$ which, according to the MCT, follows a power law $\left(T-T_{c}\right)^{\gamma}{ }^{1}$ The diffusion constant is related to the atomic mean square displacement by

$$
D=\lim _{t \rightarrow \infty} \frac{\left\langle\left|\mathbf{r}\left(t+t_{0}\right)-\mathbf{r}\left(t_{0}\right)\right|^{2}\right\rangle_{t_{0}}}{6 t} .
$$

The diffusion constants obtained, (Fig. 2), are in reasonable agreement with experiments on liquid Se. ${ }^{44,45}$ At the higher temperatures $D$ can be fitted by an Arrhenius law with an activation energy of $0.3 \mathrm{eV}$, in agreement with results from first-principle molecular dynamics simulations. ${ }^{29}$ In the inset of Fig. 2 we also show, by a dashed line, the fit by the MCT power law. Due to the correlation between $T_{c}$ and $\gamma$ such a fit can only approximately determine these values. 


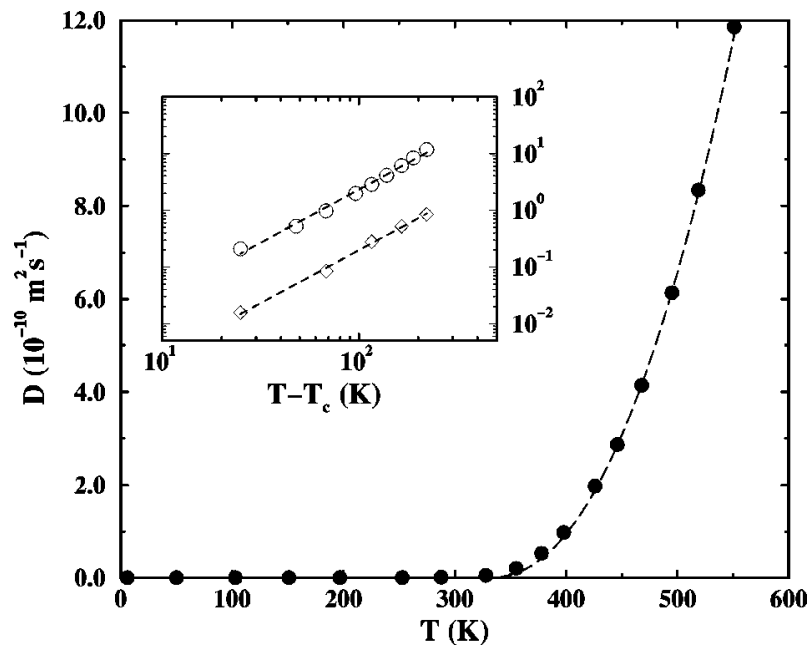

FIG. 2. Diffusion constant of liquid and amorphous Se as function of temperature. The full circles show the values obtained from the molecular dynamics simulations. The dashed line shows a fit with the power law $D \propto\left(T-T_{c}\right)^{\gamma}$. The inset shows the diffusion constant $D(\bigcirc)$ and the decay time of the $\alpha$ relaxation $\tau^{-1}(\diamond)$ vs $\left(T-T_{c}\right)$ in a $\log -\log$ representation, the $y$ axis is in units of $10^{-10} \mathrm{~m}^{2} \mathrm{~s}^{-1}$ for $D$, and $\mathrm{ps}^{-1}$ for $\tau^{-1}$.

Fixing $T_{c}$ about $10 \%$ above $T_{g}$, i.e., $T_{c}=330 \mathrm{~K}$ we get $\gamma=1.88$. From the same fit to the decay time of the $\alpha$ process (see Sec. V) we obtain for $T_{c}=330 \mathrm{~K}$ a value $\gamma$ $=1.86$, which is in excellent agreement.

\section{STRUCTURAL PROPERTIES}

Having obtained the relevant temperature scales we now turn to structural properties. First we compute the pair correlation function $(\mathrm{PCF})$ at temperatures ranging from the liquid down into the glass. The PCF is defined by

$$
g(r)=\frac{V}{4 \pi r^{2} N^{2}}\left\langle\sum_{i} \sum_{j \neq i} \delta\left(r-r_{i j}\right)\right\rangle,
$$

where $\langle\cdots\rangle$ denotes the average over configurations.

Figure 3 shows the PCF for three different temperatures, $550 \mathrm{~K}$ in the liquid phase, $290 \mathrm{~K}$ just below $T_{g}$, and $6 \mathrm{~K}$ deep in the glassy phase. The inset shows the mean coordination number. The positions of the peaks do not change strongly upon cooling, but broaden markedly. As usual oscillations at large distances are more strongly damped at high temperatures. The spatial correlations at large distances weaken with increasing temperature. In all cases the correlations are weak for distances greater than $10 \AA$. The minimum near $4 \AA$ for low temperatures signals that the second neighbor shell becomes more pronounced. The mean coordination number (Fig. 3 inset) indicates the same behavior. The mean nearest neighbor coordination is about 2.1 at all temperatures, which is similar to the experimental value. ${ }^{46}$ This value of around 2 is the signature of the chains and rings forming the amorphous selenium structure. The small excess of 0.1 compared to the ideal value of 2 indicates a prevalence of overcoordinated atoms (branching) over undercoordinated ones (chain ends). At the lowest temperature $(6 \mathrm{~K})$ we also observe a small plateau in the coordination number near $4 \AA$. The change of neighbor numbers with temperature for larger

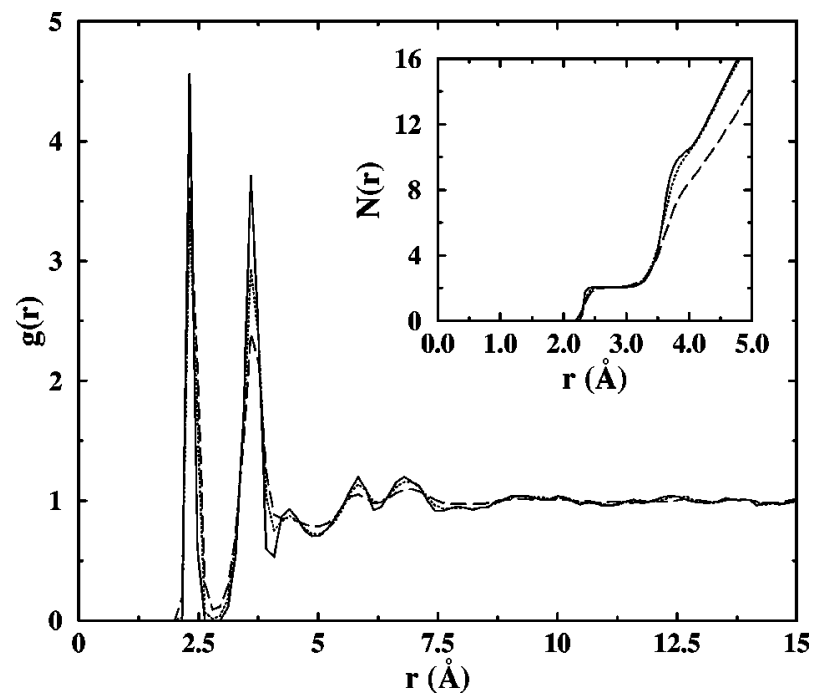

FIG. 3. Pair correlation function of Se at three different temperatures: $6 \mathrm{~K}$ (solid line), $290 \mathrm{~K}$ (dotted line), $550 \mathrm{~K}$ (long dashed line). The inset shows the mean coordination numbers for the same temperatures.

distances reflects the lower density at high temperatures. This indicates that with increasing temperature the chain structure remains, but the distance between chains increases.

From the PCF the structure factor $S(q)$ can be computed by a spatial Fourier transform

$$
S(q)=1+\frac{V}{N} \int_{0}^{\infty} 4 \pi r^{2}[g(r)-1] \frac{\sin (q r)}{q r} d r .
$$

Figure. 4 shows $S(q)$ for the three temperatures used in Fig. 3. As in the PCF, the peaks do not shift strongly with temperature, they merely become more damped with increasing temperature. The positions of the peaks agree with experiments ${ }^{46,47}$ and previous simulations on Se. ${ }^{29,48}$ In addition we see a small prepeak near $q=1 \AA^{-1}$. Experimentally the prepeak in amorphous selenium merges with the first diffraction peak at about $2 \AA^{-1}$ and is only seen as a shoulder.

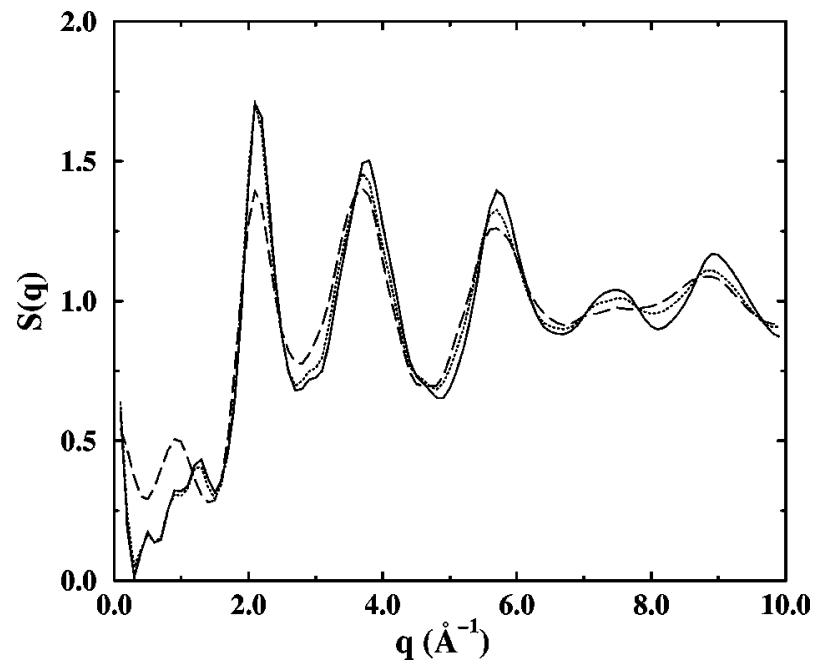

FIG. 4. Structure factor of Se at the same temperatures as Fig. 3: $6 \mathrm{~K}$ (solid line), $290 \mathrm{~K}$ (dotted line), and $550 \mathrm{~K}$ (long dashed line). 


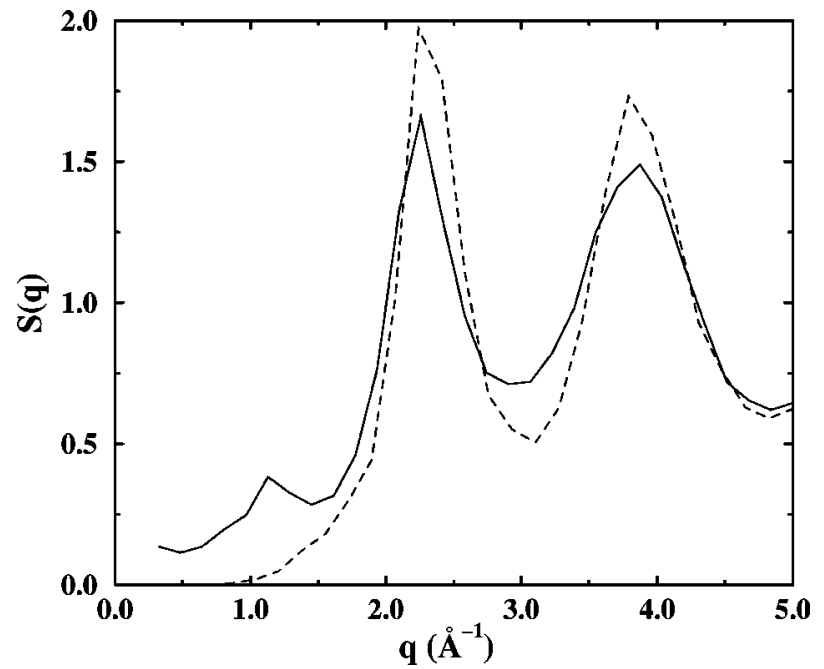

FIG. 5. Structure factor of Se at $T=0 \mathrm{~K}$ for two different densities: $\rho=4400 \mathrm{~kg} / \mathrm{cm}^{3}$ (solid line) and $\rho=5090 \mathrm{~kg} / \mathrm{cm}^{3}$ (dashed line).

To study this prepeak, we quenched two more sets of ten independent liquids, each to $0 \mathrm{~K}$ applying two different pressures: zero pressure and $10 \mathrm{GPa}$. Finally we minimized the potential energy for both sets, and released the pressure for the second set. This gave us at $T=0 \mathrm{~K}$ two sets of samples with different densities $\rho=4400 \mathrm{~kg} / \mathrm{m}^{3}$ and $\rho=5090 \mathrm{~kg} / \mathrm{m}^{3}$, both with equilibrium structures. The average potential energy per atom of the low density samples is only $3.5 \mathrm{meV}$ less than the one at the high density. This low value might indicate that at high temperature voids are present in thermodynamic equilibrium. For both sets of equilibrium structures we computed the structure factors by

$$
S(q)=\left\langle\sum_{i, j} \exp \left[i \mathbf{q}\left(\mathbf{r}_{j}(t)-\mathbf{r}_{i}(t)\right)\right]\right\rangle_{t,|\mathbf{q}|=q},
$$

where $\mathbf{q}$ are the $q$ vectors compatible with the simulation box. The minimal $q$ values, given by the periodic boundary conditions are $q=0.17 \AA^{-1}$ and $q=0.16 \AA^{-1}$ for the high and the low density samples, respectively.

Whereas the low density samples show a small prepeak near $1 \AA^{-1}$, it is absent in those of high density (Fig. 5). Checking the mean coordination number at the two densities (Fig. 6), one sees that the number of first neighbors changes very little with density: there are chains and rings at both densities. The number of second neighbors, however, is reduced for the lower density. This is the same effect as noted previously for the temperature dependence. When the density is low, i.e., when the volume is high the system is constituted of chains and rings which are further apart from each other. In other words we interpret the prepeak as the signature of correlations between holes in the structure. A similar effect was seen in simulations of $\mathrm{SiO}_{2} \cdot{ }^{49}$ As a consequence of the too high density of the crystalline structure, ${ }^{35}$ the density of our amorphous selenium is too high in comparison with the experimental value.

\section{INTERMEDIATE SELF-SCATTERING FUNCTION}

We now focus on the local relaxations. First, we compute the intermediate self-scattering function (ISSF), the correla-

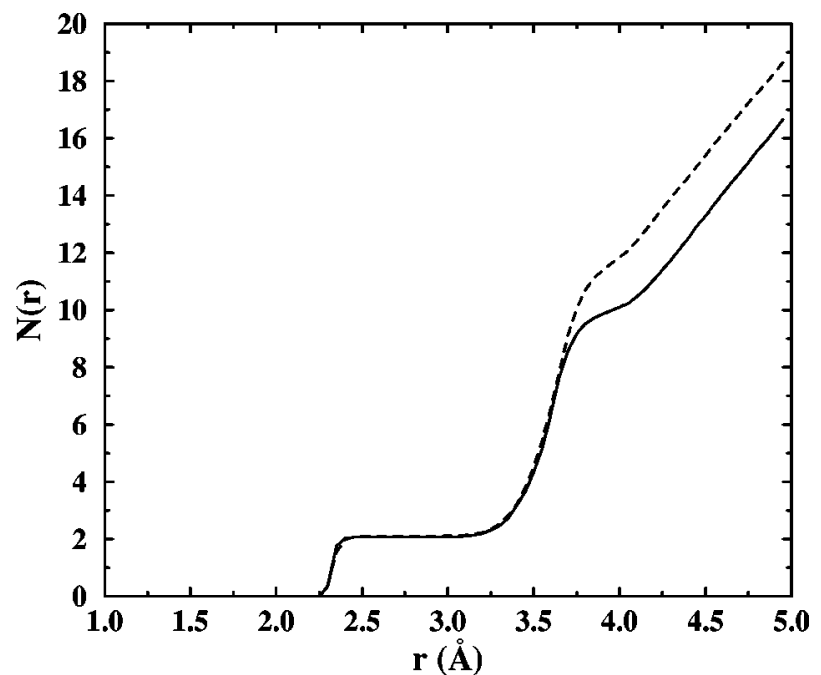

FIG. 6. Mean coordination number of $\mathrm{Se}$ at $T=0 \mathrm{~K}$ at two different densities: $\rho=4400 \mathrm{~kg} / \mathrm{cm}^{3}$ (solid line) and $\rho$ $=5090 \mathrm{~kg} / \mathrm{cm}^{3}$ (dashed line).

tion function of the density fluctuations

$$
\Phi(q, t)=\left\langle\delta \rho_{-q}\left(t+t_{0}\right) \delta \rho_{q}\left(t_{0}\right)\right\rangle_{t_{0}} .
$$

This can be rewritten as the spatial Fourier transform of the van Hove self-correlation function $G_{s}(r, t)$

$$
\Phi(q, t)=\int_{0}^{\infty} G_{s}(r, t) \frac{\sin (q r)}{q r} d r
$$

where $G_{s}(r, t)$ is given by ${ }^{50}$

$$
G_{s}(r, t)=\left\langle\delta\left(r-\left|\mathbf{r}_{i}\left(t+t_{0}\right)-\mathbf{r}_{i}\left(t_{0}\right)\right|\right)\right\rangle_{i, t_{0}} .
$$

The ISSF of Se is not easily accessible in experiment. It corresponds to the time Fourier transform of the incoherent dynamic structure factor, but neutron scattering by $\mathrm{Se}$ is mainly coherent. Nevertheless this quantity is accessible to simulation.

In Fig. 7 we report the ISSF at $q=2.1 \AA^{-1}$ correspond-

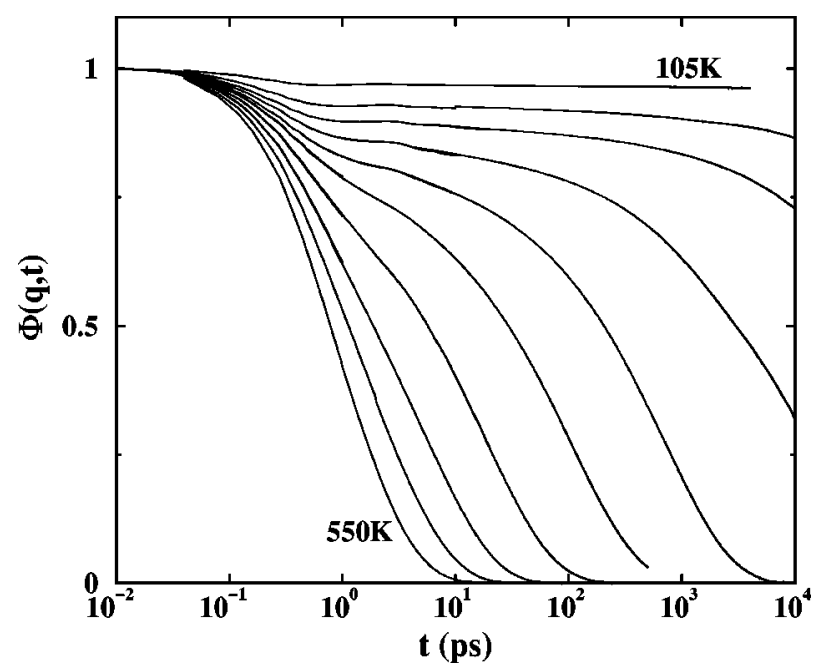

FIG. 7. ISSF of Se vs time for different temperatures above and below $T_{g}$. From top to bottom: 105, 200, 255, 290, 330, $355,400,445,495$, and $550 \mathrm{~K}$. 


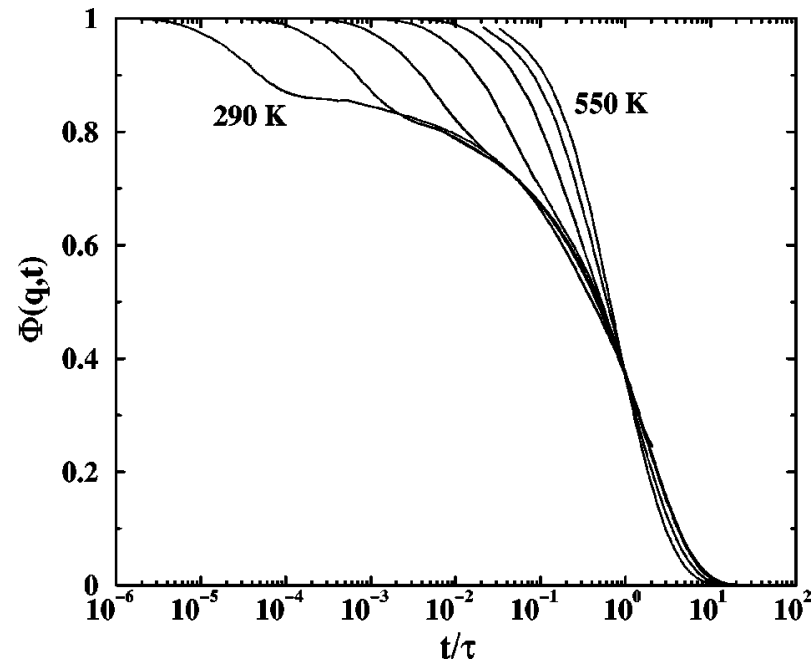

FIG. 8. Intermediate self-scattering function of Se vs the dimensionless variable $t / \tau$, where $\tau$ is defined by $\Phi(q, \tau)=1 / e$ (see inset of Fig. 2). Temperatures from left to right: 290, 330, 355, $400,445,495$, and $550 \mathrm{~K}$.

ing to the first diffraction peak of the $S(q)$ in Fig. 4. A fast decrease of $\Phi(q, t)$ on the time scale of a picosecond is observed at all temperatures. When decreasing the temperature a shoulder appears for intermediate time scales. As the system approaches the glass transition temperature, $\Phi(q, t)$ starts to saturate and finally shows a plateau for intermediate and long times. As customary this curve is described as follows: First there is the ballistic or vibrational regime (corresponding to the fast decreases at low time). Then, for $T>T_{c}, \Phi(q, t)$ goes to zero (the so-called $\alpha$ regime). The shoulder or plateau at lower $T$ corresponds to the so-called $\beta$ regime. This plateau indicates that the system is trapped in a limited area of phase space.

According to the MCT, the $\alpha$ regime above $T_{c}$ is driven by a master curve which is obtained by rescaling the time by a characteristic decay time $\tau$ defined as the time when the ISSF drops to $1 / e, \Phi(q, \tau)=1 / e$. Above $T_{c}$, similar to the diffusion constant $D$, these values $\tau(T)$ should obey a power law $\tau(T)=\left(T-T_{c}\right)^{-\gamma}$. Fixing $T_{c}=330 \mathrm{~K}$ we get a good fit with $\gamma=1.86$, (see inset in Fig. 2). Using this $\tau$ the master curve can be written in scaled form as ${ }^{2}$

$$
\Phi(q, t / \tau)=\Phi_{0} \exp \left[-(t / \tau)^{\beta}\right] .
$$

Figure 8 presents the curves for temperatures above $T_{g}$ rescaled by their respective $\tau$. We get a value $\beta=0.53$ for temperatures ranging from $T=330 \mathrm{~K}$ to $T=430 \mathrm{~K}$. We do not intend to give an elaborate test of the MCT but show the rescaled curves merely to indicate that the rescaling procedure approximately holds.

The most striking effect is the plateau corresponding to the $\beta$ regime. It indicates that the system falls out of equilibrium, and that atoms are trapped in a well of the energy landscape. This poses the question of whether all the atoms are affected equally or not.

The same question can be asked for the $\alpha$ regime, which can be represented by a stretched exponential decay law. Such a law can either mean that a stretched exponential decay law governs the whole system, or it originates from an

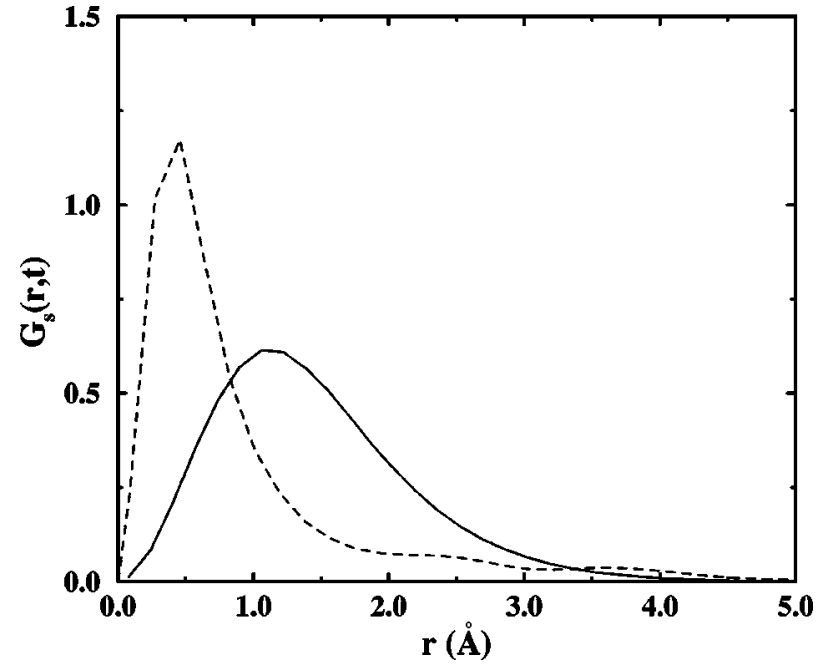

FIG. 9. Van Hove correlation function of Se at two different temperatures (solid line: $550 \mathrm{~K}$; dashed line $255 \mathrm{~K}$ ) and times, $t$ $=2.1 \mathrm{ps}$ for $550 \mathrm{~K}$ and $t=36 \mathrm{~ns}$ for $255 \mathrm{~K}$, respectively. The different times reflect the higher mobility (or diffusion) in the liquid.

average of normal exponential laws with different time constants. The first picture is a homogeneous scenario (the system is the same everywhere) and the second one is heterogeneous.

\section{HETEROGENEITY OR HOMOGENEITY?}

To answer this question, we again use the van Hove correlation function (VHF) which represents the probability for an atom to move a distance $r$ during a time $t$. If all the atoms have the same mobility the VHF is a Gaussian multiplied by the geometrical factor $4 \pi r^{2}$. In the following this factor is always thought to be included when we speak of Gaussianity. In the opposite case if some atoms are trapped or some are more mobile than the majority the VHF will, in general, be non-Gaussian. As an example in Fig. 9 we show the VHF for two different temperatures for the same length range but for two different times. Obviously at high temperatures the system has a higher diffusion constant and the atoms will move faster over a given distance. More striking is that at high temperatures (in the liquid) the VHF has a Gaussian shape, whereas at low temperatures (in the glass) the VHF has an extended tail and cannot be described by a Gaussian. Some atoms move over much larger distances than the average atom, i.e., they have a much higher mobility.

In order to quantify these findings, and in accordance with previous work, we introduce the non-Gaussianity parameter (NGP) $\alpha_{2}$ (Ref. 51)

$$
\alpha_{2}(t)=\frac{3 \mu_{4}}{5 \mu_{2}^{2}}-1,
$$

where $\mu_{2}$ and $\mu_{4}$ are the second and fourth moments of the VHF, $\mu_{2}=\left\langle r^{2}(t)\right\rangle$, and $\mu_{4}=\left\langle r^{4}(t)\right\rangle$, respectively. The NGP is identical to zero for a Gaussian VHF.

Figure 10 shows, in a log-linear representation, the $\alpha_{2}$ at different temperatures from the liquid to the glass for a time span covering 6 decades. At very short times the NGP is nearly zero at all temperatures. The limiting behavior for 


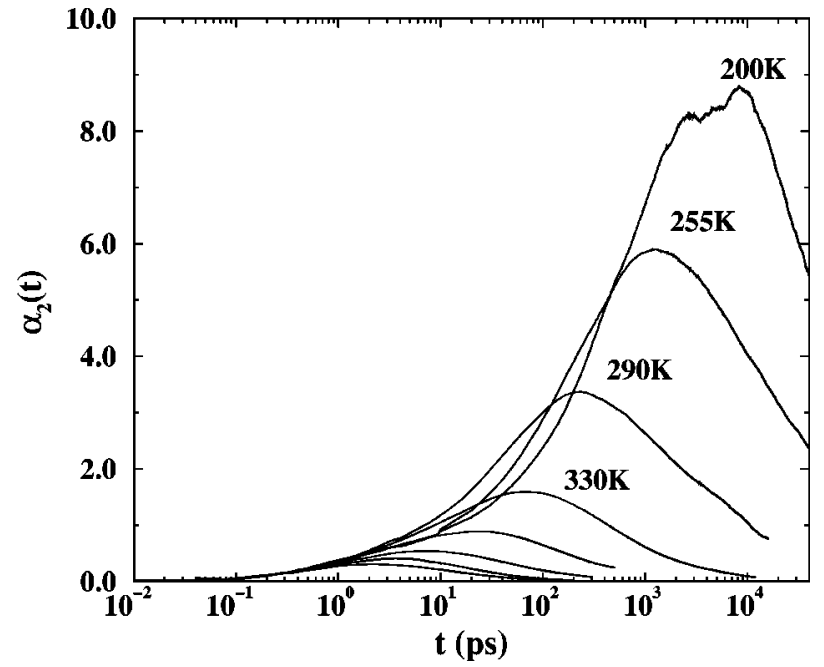

FIG. 10. Log-linear plot of the non-Gaussianity parameter $\alpha_{2}$ of Se vs time for several temperatures. From top to bottom: 200, 255, 290, 330, 355, 400, 445, and $495 \mathrm{~K}$.

large times is more difficult to observe, especially at low temperatures. Nevertheless the NGP clearly tends to zero. Furthermore, all the curves below and above the glass transition scale to the same curve in the short time range as already shown by Kob and Andersen in their study of a binary supercooled Lennard-Jones liquid. ${ }^{21}$ Our work shows that this property persists in the glassy phase and for a completely different structure.

In the intermediate time range the NGP has a maximum indicating heterogeneity. At high temperatures, in the liquid above $T_{c}$, this maximum is small and located around $10 \mathrm{ps.}$ Upon cooling, it slowly moves to higher times. For the temperatures below $T_{c}$, the behavior is different. The value of the maximum is larger than 2 and it grows by a factor of 2 upon cooling by $50 \mathrm{~K}$. Similarly the position of the maximum shifts by about an order of magnitude for each $50 \mathrm{~K}$. These two observations suggest that as the system is cooled, especially below $T_{g}$, the non-Gaussianity becomes more and more pronounced at intermediate time scales.

We now focus on the short time behavior at very low temperatures. In Fig. 11, we present the evolution of the NGP for two different samples A and B (out of our four different samples) at a very low temperature $T=6 \mathrm{~K}$. The inset gives the same curves in linear-linear representation to show them clearly at intermediate times. The curves coincide during the first picosecond in the vibrational regime. For the larger, intermediate time scale the NGP of sample A [Fig. 11(a)] oscillates around a value of 0.2 , but the one of sample B [Fig. 11(b)] rises. The two other samples behave similarly to sample A. What is the reason for this difference in the non-Gaussian behavior of these two kinds of samples? The evolution of both total energy and volume were equivalent. The mean square displacements, however, evolve differently (Fig. 12). In sample A [Fig. 12(a)] it oscillates around a mean value during the entire simulation run, whereas it shows steps for sample B [Fig. 12(b)]. Thus, while sample A stays in one well of the energy landscape, sample B moves from one well into another. We can identify at least two different wells for sample B. We conclude that the value $\alpha_{2} \approx 0.2$ of the NGP corresponds to the vibrations in the

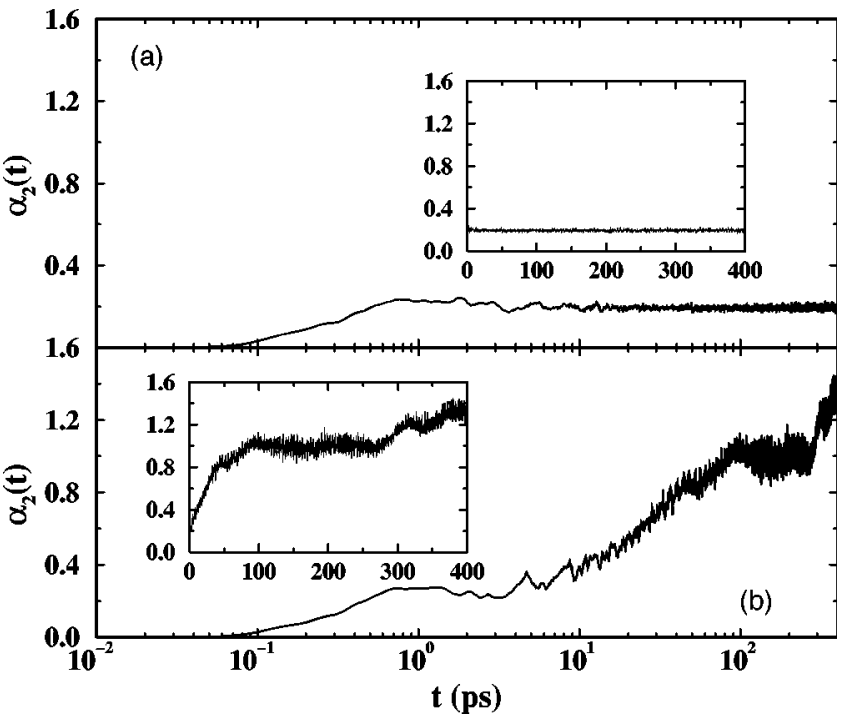

FIG. 11. Log-linear plot of the non-Gaussianity parameter of Se vs time for two samples (A and B) [(a) and (b), respectively] at low temperature: $6 \mathrm{~K}$. The insets show the same quantities in a linearlinear plot.

liquid and amorphous selenium. Relaxations from one minimum of the energy landscape to another lead to an increase in the NGP. It has been shown previously that these local relaxations are collective jumps of ten and more atoms. ${ }^{37}$ The jump length of a single atom is much less than the nearest neighbor distance. The different behavior of the samples, shown in Fig. 12, reflects the low probability for relaxations at low temperature. It is not restricted to Se but is typical for glasses. The same has also been reported from a simulation of simple soft sphere glass ${ }^{52}$ and is observed experimentally in the telegraph noise of the electric resistivity of point contacts. ${ }^{53}$

\section{CONCLUSION}

In this paper we have presented results of a molecular dynamics simulation on the structure and relaxations of liq-

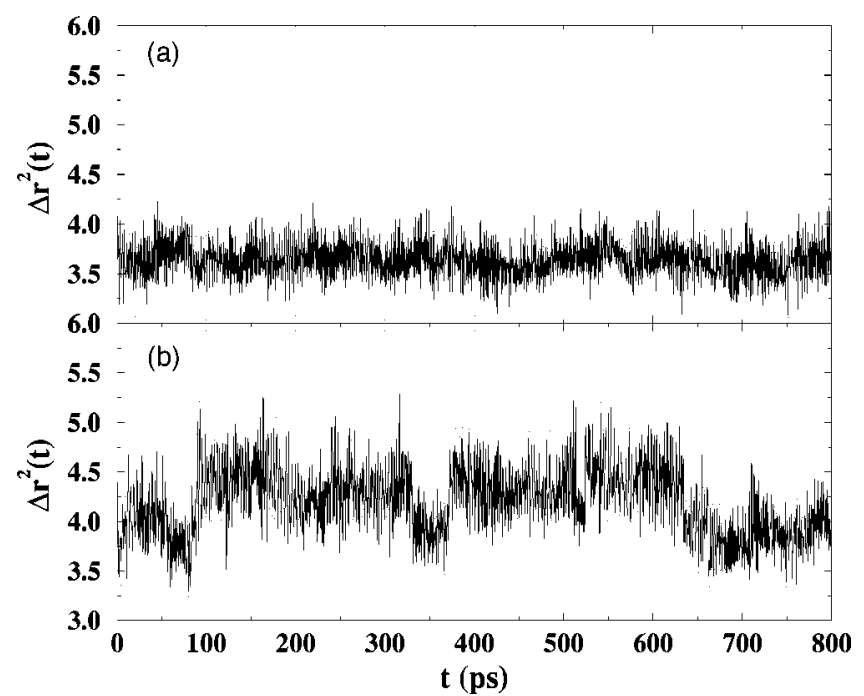

FIG. 12. Mean square displacement for samples A (top) and B (bottom) during the simulation. 
uid and amorphous Se. We determined the glass transition and critical temperatures, the pair correlation function, and the structure factor. From the pair correlation function, and in agreement with experiments, we concluded that both liquid and amorphous selenium are constituted of chains and rings with a mean coordination number of 2.1, slightly above the ideal value 2 . Rings and chains are interconnected. The structure factor shows a small prepeak around $1 \AA^{-1}$, which in experiment is shown only as a shoulder of the main peak. This prepeak is explained in terms of a correlation of voids between the selenium chains. To prove this assumption we computed the structure factors of two sets of samples with two different densities. At the higher density no prepeak is observed.

The van Hove correlation function was calculated and utilized to compute the intermediate scattering function and the non-Gaussianity parameter. For the intermediate selfscattering function, the time correlation of the density fluctuation, we find the classical behavior: at short times a rapid decrease corresponding to the ballistic (or vibrational) regime, and at long times a slow decay corresponding to the $\alpha$ regime. When the system reaches $T_{c}$ a shoulder and below $T_{g}$ a plateau evolves between these two regimes. This corresponds to the $\beta$ regime, and to a memory effect of the correlation function, in other words the system falls out of equilibrium.
The non-Gaussianity parameter shows that at both short and long times the relaxations are homogeneous or only weakly inhomogeneous and all the atoms are largely equivalent. For the intermediate time range (corresponding to the $\beta$ regime) NGP depends on the temperature. The lower the temperature the higher the NGP, i.e., the higher the heterogeneity. At low temperatures the increase of non-Gaussianity is due to relaxations. We conclude the following scenario for the heterogeneity: at all temperatures both above and below $T_{g}$ there is a small increase of heterogeneity $\left(\alpha_{2}=0.2\right)$ due to vibrations at short time, at intermediate times a pronounced increase, due to the relaxations especially at temperatures below $T_{g}$, and finally a decrease due to long range diffusion (flow motion). These different regimes correspond to the different regimes observed in the intermediate selfscattering function $\Phi(q, t)$. This scenario seems to be common to different materials.

\section{ACKNOWLEDGMENTS}

We would like to thank C. Oligschleger for her help at the beginning of this work. We also thank M. Kluge, J. Matsui, and U. Buchenau for fruitful and exciting discussions. One of us (D.C.) is grateful to the A. von Humboldt foundation for financial support.
${ }^{1}$ W. Götze, in Liquids, Freezing and the Glass Transition, edited by J.-P. Hansen, D. Leveque, and J. Zinn-Justin (North-Holland, New York, 1991).

${ }^{2}$ J.-L. Barrat, in Physics of Glasses: Structure and Dynamics, AIP Conf. Proc. No. 489, edited by P. Jund and R. Jullien (AIP, New York, 1999).

${ }^{3}$ F. Mezei, W. Knaak, and B. Farago, Phys. Rev. Lett. 58, 571 (1994).

${ }^{4}$ C. Alba-Simionescu and M. Krauzman, J. Chem. Phys. 102, 6574 (1995)

${ }^{5}$ D. Richter, R. Frick, W. Knaak, and B. Farago, Phys. Rev. Lett. 61, 2465 (1988).

${ }^{6}$ Y. Scheyer, C. Levelut, J. Pelous, and D. Durand, Phys. Rev. B 57, 11212 (1998).

${ }^{7}$ W. Kob and H.C. Andersen, Phys. Rev. E 52, 4134 (1995).

${ }^{8}$ H. Teichler, Phys. Rev. E 53, 4287 (1996).

${ }^{9}$ R. Yamamoto and A. Onuki, Phys. Rev. E 58, 3515 (1998).

${ }^{10}$ F. Affouard and M. Descamps, Phys. Rev. B 59, 9011 (1999).

${ }^{11}$ A. Arbe and J. Colmenero, Phys. Rev. Lett. 81, 590 (1998).

${ }^{12}$ J. Colmenero, A. Arbe, A. Alegría, M. Monkenbusch, and D. Richter, J. Phys.: Condens. Matter 11, A363 (1999).

${ }^{13}$ M.T. Cicerone, F.R. Blackburn, and M.D. Ediger, J. Chem. Phys. 102, 471 (1995).

${ }^{14}$ G. Williams and J. Fournier, J. Chem. Phys. 104, 5690 (1996).

${ }^{15}$ R. Zorn, Phys. Rev. B 55, 6249 (1997).

${ }^{16}$ T. Kanaya, I. Tsukushi, and K. Kaji, Prog. Theor. Phys. Suppl. 126, 133 (1997).

${ }^{17}$ B. Doliwa and A. Heuer, Phys. Rev. Lett. 80, 4915 (1998).

${ }^{18}$ B. Doliwa and A. Heuer, J. Phys.: Condens. Matter 11, A277 (1999).

${ }^{19}$ H. Miyagawa and Y. Hiwatari, Phys. Rev. A 40, 6007 (1989).
${ }^{20}$ T. Ogadaki and J. Matsui, in Slow Dynamics in Complex Systems, AIP Conf. Proc. No. 469, edited by M. Tokuyama and I. Oppenheim (AIP, New York, 1999).

${ }^{21}$ W. Kob and H.C. Andersen, Phys. Rev. E 51, 4626 (1995).

${ }^{22}$ M. Dzugutov, Phys. Rev. A 46, R2984 (1992).

${ }^{23}$ R. DiLeonardo, L. Angelani, G. Parisi, and G. Ruocco, cond-mat/0001311 (unpublished).

${ }^{24}$ P. Andonov, J. Non-Cryst. Solids 47, 297 (1982).

${ }^{25}$ B.W. Corb, W.D. Wei, and B.L. Averbach, J. Non-Cryst. Solids 53, 29 (1982).

${ }^{26}$ M. Misawa and K. Suzuki, J. Phys. Soc. Jpn. 44, 1612 (1978).

${ }^{27}$ D. Hohl and R.O. Jones, Phys. Rev. B 43, 3856 (1991).

${ }^{28}$ C. Bichara, A. Pellegati, and J.-P. Gaspard, Phys. Rev. B 49, 6581 (1994); C. Bichara, J.-Y. Raty, and J.-P. Gaspard, J. NonCryst. Solids 250, 419 (1999).

${ }^{29}$ F. Kirchhoff, M.J. Gillan, J.M. Holender, G. Kresse, and J. Hafner, J. Phys.: Condens. Matter 8, 9353 (1996); F. Kirchhoff, G. Kresse, and M.J. Gillan, Phys. Rev. B 57, 10482 (1998).

${ }^{30}$ F. Shimitsu, H. Kaburaki, T. Oda, and Y. Hiwatari, J. Non-Cryst. Solids 250, 433 (1999).

${ }^{31}$ T. Yamaguchi, H. Ohtani, and F. Yonezawa, J. Non-Cryst. Solids 250, 437 (1999).

${ }^{32}$ N.G. Almarza, E. Enciso, and F.J. Bermejo, Europhys. Lett. 17, 595 (1992); M. Garcia-Hernández, B. Fåk, J.L. Martínez, E. Enciso, N.G. Almarza, and A. Criado, Phys. Rev. B 48, 149 (1993); F.J. Bermejo, E. Enciso, A. Criado, J.L. Martínez, and M. Garcia-Hernández, ibid. 49, 8689 (1994); F.J. Bermejo, M. Garcia-Hernández, T. Mason, J.L. Martínez, E. Enciso, and A. Criado, ibid. 50, 13286 (1994); F.J. Bermejo, M. GarciaHernández, F.J. Mompeán, D. MacMorrow, and J.L. Martínez, ibid. 51, 11932 (1995). 
${ }^{33}$ S. Balalasubramian, K.V. Damodaran, and K.J. Rao, Chem. Phys. 166, 131 (1992).

${ }^{34}$ F.H. Stillinger, T.A. Weber, and R.A. LaViolette, J. Chem. Phys. 85, 6460 (1986).

${ }^{35}$ C. Oligschleger, R.O. Jones, S.M. Reimann, and H.R. Schober, Phys. Rev. B 53, 6165 (1996).

${ }^{36}$ C. Oligschleger and H.R. Schober, Physica A 201, 391 (1993); C. Oligschleger and J.C. Schön, J. Phys.: Condens. Matter 9, 1049 (1997).

${ }^{37}$ C. Oligschleger and H.R. Schober, Solid State Commun. 93, 1031 (1995); H.R. Schober, C. Gaukel, and C. Oligschleger, Prog. Theor. Phys. Suppl. 126, 67 (1997).

${ }^{38}$ U. Buchenau, Yu. Galperin, V.L. Gurevich, and H.R. Schober, Phys. Rev. B 43, 5039 (1991).

${ }^{39}$ T. Koslowski, Z. Phys. Chem. (Munich) 210, 45 (1999).

${ }^{40}$ M. Parrinello and A. Rahman, Phys. Rev. Lett. 45, 1196 (1980).

${ }^{41}$ M. Parrinello and A. Rahman, J. Appl. Phys. 52, 7182 (1981).

${ }^{42}$ W.C. Swope, H.C. Andersen, P. Berens, and K.R. Wilson, J.
Chem. Phys. 76, 637 (1982).

${ }^{43}$ U. Buchenau and R. Zorn, Europhys. Lett. 18, 523 (1992).

${ }^{44}$ W.A. Philips, U. Buchenau, N. Nücker, A.-J. Dianoux, and W. Petry, Phys. Rev. Lett. 63, 2381 (1989).

${ }^{45}$ A. Axmann, W. Gissler, A. Kollmar, and T. Springer, Discuss. Faraday Soc. 50, 74 (1970).

${ }^{46}$ M. Edeling and W. Freyland, Ber. Bunsenges. Phys. Chem. 85, 1049 (1981).

${ }^{47}$ K. Tamura and S. Hosokawa, Ber. Bunsenges. Phys. Chem. 96, 681 (1992).

${ }^{48}$ N.G. Almarza, E. Enciso, and F.J. Bermejo, J. Chem. Phys. 99, 6876 (1993).

${ }^{49}$ S.R. Elliott, J. Non-Cryst. Solids 182, 40 (1995).

${ }^{50}$ L. van Hove, Phys. Rev. 95, 249 (1954).

${ }^{51}$ A. Rahman, Phys. Rev. 136, A405 (1964).

${ }^{52}$ C. Oligschleger and H.R. Schober, Phys. Rev. B 59, 811 (1999).

${ }^{53}$ V.I. Kozub and C. Oligschleger, J. Phys.: Condens. Matter 10, 8033 (1998). 